01

\title{
Измерение напряженности электрического поля СВЧ излучения на частоте радиационного перехода между ридберговскими состояниями атомов ${ }^{85} \mathrm{Rb}$
}

\author{
(С) Е.Ф. Стельмашенко ${ }^{1}$, О.А. Клезович ${ }^{1}$, В.Н. Барышев ${ }^{1}$, В.А. Тищенко ${ }^{1}$, И.Ю. Блинов ${ }^{1}$, \\ В.Г. Пальчиков ${ }^{1,2}$, В.Д. Овсянников ${ }^{1,3}$ \\ ${ }^{1}$ ФГУП „ВНИИФТРИ“, \\ 141570 Менделеево, Московская обл., Россия \\ ${ }^{2}$ Национальный исследовательский ядерный университет „МИФИ“, \\ 115409 Москва, Россия \\ ${ }^{3}$ Воронежский государственный университет, \\ 394018 Воронеж, Россия \\ e-mail:stelmashenko@vniiftri.ru
}

Поступила в редакцию 05.02.2020 г.

В окончательной редакции 17.02.2020 г.

Принята к публикации 10.03.2020 г.

Определены спектральные характеристики резонансного эффекта электромагнитно-индуцированной (ЭИП) прозрачности атомарных паров рубидия, индуцируемого интенсивным излучением с длиной волны в области от 479 до $486 \mathrm{~nm}$ в присутствии сверхвысокочастотного (СВЧ) излучения на частоте от 1 до $300 \mathrm{GHz}$, расщепляющего энергию перехода атома из резонансного $5 P_{3 / 2}$-состояния в ридберговское $n D_{5 / 2}$-состояние. Величина расщепления пика ЭИП пропорциональна напряженности электрического поля СВЧ излучения. Получены простые аппроксимационные выражения для численного расчета амплитуды перехода между ридберговскими $n D_{5 / 2^{-}}$и $(n+1) P_{3 / 2}$-состояниями с большими главными квантовыми числами $n$. Разработана установка для измерения напряженности электрического поля СВЧ излучения на основе измерения частоты расщепления резонанса ЭИП в спектре поглощения пробного излучения.

Ключевые слова: СВЧ излучение, напряженность электрического поля, электромагнитно-индуцированная прозрачность (ЭИП), расщепление Аутлера-Таунса, ридберговские состояния атомов.

DOI: $10.21883 /$ OS.2020.08.49698.25-20

\section{Введение}

В настоящее время перед международным метрологическим сообществом стоит цель сделать любые измерения количественно выраженными в единицах Международной системы единиц (СИ). Измерения физических величин на основе квантовых эффектов в атомах непосредственно обеспечивают такую прослеживаемость к СИ. Примером такого нового подхода к прослеживаемым к СИ измерениям являются интенсивно ведущиеся исследования по созданию широкополосного измерителя напряженности электрического поля сверхвысокочастотного (СВЧ) электромагнитного излучения, основанного на взаимодействии СВЧ излучения с атомами щелочных металлов, возбужденных до ридберговских состояний. Наиболее последовательно этот подход изложен в работах $[1,2]$. В них определены потенциальные возможности квантового измерителя напряженности электрического поля (НЭП) и области возможного применения, среди которых важнейшими отмечены - эталонирование напряженности электрического поля в широком частотном диапазоне СВЧ излучения и визуализация электрического поля с высоким субмиллиметровым пространственным разрешением. Отмечается, что, не внося значительных изменений в конфигурацию экспериментальной установки, можно с ее помощью измерять НЭП в диапазоне частот от нескольких десятков $\mathrm{MHz}$ до $500 \mathrm{GHz}$ и выше. К настоящему времени проведены измерения НЭП в диапазоне от 0.8 до $1000 \mathrm{~V} / \mathrm{m}$ [3]. По сравнению с существующими, базирующимися на антенной технике, методами квантово-оптический метод измерения НЭП обладает более высокой чувствительностью, ограниченной пределом дробовых шумов фотодетектирования $5 \mu \mathrm{Vcm}^{-1} \mathrm{~Hz}^{-1}[4]$, и высокой точностью с $0.5 \%$ неопределенностью измерения величины напряженности поля в несколько десятков V/m [5].

Целью представляемой нами работы является теоретический и экспериментальный анализы эффекта расщепления оптического резонанса электромагнитноиндуцированной прозрачности (ЭИП) в СВЧ поле, разработка прибора для измерения НЭП, отработка техники измерения НЭП на нескольких дискретных частотах в широком 2-200 GHz диапазоне.

НЭП СВЧ излучения можно измерить с помощью эффекта Аутлера-Таунса (АТ) [6] на электродипольных переходах между ридберговскими $n D_{5 / 2^{-}}$и $(n+1) P_{3 / 2^{-}}$ состояниями атомов рубидия $[1,7,8]$. Частоты таких переходов занимают широкий диапазон от сотен мегагерц (для $n>150$ ) до десятых долей и единиц терагерц (для $\mathrm{n}<20$ ), открывая возможность найти резонансный 
дипольный переход практически для любой частоты СВЧ излучения $\omega=E_{n L_{j}}-E_{n^{\prime} L^{\prime},}+\varepsilon$ (в работе, если не оговорено специально, используется атомная система единиц $(e=\hbar=m=1)$. Здесь $\varepsilon-$ расстройка резонанса $(|\varepsilon| \ll \omega)$ относительно частоты перехода между ридберговскими состояниями $\left|n L_{j}\right\rangle$ и $\left|n^{\prime} L_{j^{\prime}}^{\prime}\right\rangle$ с энергиями $E_{n L_{j}}$ и $E_{n^{\prime} L_{j^{\prime}}^{\prime}}$ невозмущенного атома. СВЧ излучение индуцирует расщепление АТ резонансных состояний на два близких уровня с энергиями, определяемыми решением секулярного уравнения для вырожденных квазиэнергетических состояний [9]:

$$
E_{n L_{j}\left(n^{\prime} L_{j^{\prime}}^{\prime}\right)}^{ \pm}=E_{n L_{j}\left(n^{\prime} L_{j^{\prime}}^{\prime}\right)} \pm \Delta E(\varepsilon, E),
$$

где

$$
\Delta E(\varepsilon, E)=\frac{1}{2} \sqrt{\varepsilon^{2}+\Omega^{2}}
$$

представляет собой решение секулярного уравнения для двукратно вырожденных состояний, а частота Раби $\Omega=\mathscr{R} E$ определяется произведением матричного элемента $\mathscr{R}=\left\langle n L_{j}|z| n^{\prime} L_{j^{\prime}}^{\prime}\right\rangle$ дипольного перехода между резонансными состояниями и напряженности $E$ электрического поля СВЧ излучения $(z-$ проекция оператора электрического дипольного момента на направление вектора поляризации излучения). При точном резонансе $(\varepsilon=0)$ расщепление $\Delta f=E_{n L_{j}}^{+}-E_{n L_{j}}^{-}=|\Omega|$ совпадает с частотой Раби. Таким образом, измерив расщепление ридберговского состояния $\Delta f$ при точном СВЧ резонансе, можно определить напряженность электрического поля резонансного излучения из выражения

$$
E=\frac{\Delta f}{|\mathscr{R}|} \text {. }
$$

В настоящей работе выполнены экспериментальные измерения расщепления $\Delta f$ и проведены теоретические расчеты матричных элементов $\mathscr{R}$ электродипольных переходов из $n D_{5 / 2}$-состояний в состояния $(n+1) P_{3 / 2}$ в атомах рубидия на частотах СВЧ излучения $\omega=E_{n D_{5 / 2}}-E_{(n+1) P_{3 / 2}}$ от 2.1 до $196.55 \mathrm{GHz}$, соответствующих переходам между ридберговскими состояниями с главными квантовыми числами $n$ от 23 до 99. Для измерения $\Delta f$ используется эффект ЭИП атомарной среды для пробного лазерного излучения на частоте линии резонансного поглощения нормального атома с длиной волны $\lambda_{p}=780 \mathrm{~nm}[1,7,8,10]$. Для этого атомы рубидия, локализованные в стеклянной ячейке, подвергают воздействию достаточно интенсивного связывающего линейно поляризованного лазерного излучения с длиной волны $\lambda_{c}=479-486 \mathrm{~nm}$, способного возбудить атомы из резонансного $5 P_{3 / 2}$-состояния в ридберговское состояние $n D_{5 / 2}$ с большим главным квантовым числом $n>20$.

\section{Расчет амплитуды дипольного перехода $n D_{5 / 2} \rightarrow(n+1) P_{3 / 2}$}

Матричный элемент $\mathscr{R}$ дипольного радиационного перехода ридберговского электрона может быть рас- считан в одноэлектронном приближении стандартными методами атомной спектроскопии $[9,11]$. Полагая ось $z$ направленной вдоль общей оси квантования проекций полных моментов основного, резонансного и ридберговских состояний, по которой направлены векторы поляризации всех трех линейно поляризованных излучений - пробного, связывающего и СВЧ. Тогда магнитные квантовые числа ридберговских состояний сохраняют численное значение проекции основного состояния $m= \pm 1 / 2$. Вычисляя интегралы по угловым переменным ридберговского электрона стандартными методами квантовой теории углового момента [12], получим

$$
\mathscr{R}=\frac{\sqrt{6}}{5}\left\langle R_{n D_{5 / 2}}(r)|r| R_{(n+1) P_{3 / 2}}(r)\right\rangle \text {. }
$$

Расчет радиального матричного элемента $\left\langle R_{n D_{5 / 2}}(r)|r| R_{n+1) P_{3 / 2}}(r)\right\rangle \quad$ в этом выражении можно выполнить одним из хорошо апробированных полуэмпирических методов - методом модельного потенциала Фьюса (МПФ) или методом квантового дефекта (МКД) [9,11,13]. В обоих методах радиальные волновые функции записываются в виде полиномов по степеням аргумента:

$$
\begin{gathered}
R_{n l}^{(\mathrm{MПФ)}}(r)=\frac{2 Z^{3 / 2}}{v_{n l}^{2}} \sqrt{\frac{n_{r} !}{\Gamma\left(v_{n l}+\lambda+1\right)}} \exp \left(-\frac{x}{2}\right) x^{\lambda} L_{n_{r}}^{2 \lambda+1}(x), \\
R_{n l}^{(\text {МКД })}(r)=\frac{2 Z^{3 / 2}}{v_{n l}^{2} \sqrt{\Gamma\left(v_{n l}+l+1\right)}} \frac{W_{v_{n l} l+1 / 2}(x)}{x},
\end{gathered}
$$

где $x=2 Z r / v_{n l}$ включает в себя радиальную переменную $r$ и эффективное главное квантовое число $v_{n l}=Z / \sqrt{-2 \varepsilon_{n l}} ; \quad \lambda=v_{n l}-n_{r}-1 \quad$ и $\quad n_{r}=v_{n l}-\lambda-1=$ $=0,1,2, \ldots$ - эффективное орбитальное и радиальное квантовые числа.

$$
L_{n_{r}}^{2 \lambda+1}(x)=\frac{(2 \lambda+1)_{n_{r}}}{n_{r} !} \sum_{k=0}^{n_{r}} \frac{\left(-n_{r}\right)_{k}}{k !(2 \lambda+2)_{k}} x^{k}
$$

— присоединенный полином Лагерра, порядок которого определяет индекс $n_{r}$.

$$
W_{v_{n l} l+1 / 2}(x)=\exp \left(-\frac{x}{2}\right) x^{v_{n l}} \sum_{k=0}^{\left[v_{n l}\right]} \frac{\left(v_{n l}+l+1\right)_{k}\left(v_{n l}-l\right)_{k}}{k ! x^{k}}
$$

- функция Уиттекера [14]. Следует отметить, что число слагаемых в суммах (6) и (7) может различаться, поскольку целая часть главного квантового числа $\left[v_{n l}\right]$ для состояний с большим орбитальным моментом может существенно превосходить радиальное квантовое число $n_{r}$. Это означает, что наборы слагаемых с низшими степенями радиальной переменной в функциях $R_{n l}^{(\mathrm{MПФ)}}(r)$ и $R_{n l}^{(\text {МКД) }}(r)$ в (5) могут быть разными. При этом показатели старших степеней аргументов здесь одинаковы, поскольку $\lambda+n_{r}=v_{n l}-1$. Соответствующие коэффициенты при совпадении эффективного 
орбитального квантового числа $\lambda$ с орбитальным моментом $l$ становятся одинаковыми. Таким образом, функции МПФ и МКД на малых расстояниях $r$ от атомного ядра различаются, а на больших расстояниях они практически одинаковы. Поэтому численные значения матричных элементов дипольных переходов между близкими по энергии состояниями, рассчитанные с функциями МПФ и МКД, практически совпадают друг с другом. Согласие улучшается с ростом орбитального момента $l$.

С использованием функций (5) интегрирование по радиальной переменной можно выполнить аналитически, так что радиальный матричный элемент можно представить в виде линейной комбинации гипергеометрических функций, расчеты которых можно выполнить с любой точностью с помощью имеющихся в настоящее время вычислительных средств. При этом численные значения параметров функций (5) определяются по спектру уровней $D_{5 / 2}$ и $P_{3 / 2}$ серий атома рубидия (см., например, таблицы энергетических уровней $[15,16])$.

Результаты численных расчетов матричных элементов (4) дипольного перехода $n D_{5 / 2} \rightarrow(n+1) P_{3 / 2}$ в области главных квантовых чисел $n=10-200$ ридберговских состояний атомов рубидия хорошо согласуются с соответствующими данными расчетов в работе [1]. Эти данные удобно аппроксимировать квадратным полиномом, полученным стандартной процедурой интерполяции. В частности, интерполяция по численным значениям, рассчитанным в МКД-приближении для состояний с $n=30,60,100$ дает

$$
\mathscr{R}(n)=0.65885 n^{2}-1.6213 n-0.28161 .
$$

Это выражение воспроизводит численные значения расчетов с функциями $R_{n l}^{(\text {МКД) }}(r)$ в области главных квантовых чисел $n>10$ с относительной погрешностью не более $10^{-6}$. Аналогичную аппроксимацию можно получить из расчетов методом МПФ, при этом коэффициент при $n^{2}$ соответствующего полинома в точности совпадает с коэффициентом полинома (8), а отличие получаемых значений $\mathscr{R}(n)$ не превышает $0.1 \%$.

\section{Измерение напряженности поля СВЧ излучения на частоте $n D_{5 / 2} \rightarrow(n+1) P_{3 / 2}$-перехода}

Вследствие однозначного идейного характера и экспериментальной простоты квантово-оптического метода измерения напряженности $E$-поля схема нашей экспериментальной установки не отличается принципиально от схем, примененных в работах других исследовательских групп. Различия могут касаться выбора типов газовых ячеек с парами щелочных атомов, типов лазерных источников и способов их частотной модуляции и стабилизации, способов регистрации сигналов ЭИП и определения частоты расщепления сигнала ЭИП. В проведенных нами экспериментах использовалась стеклянная (пирекс) ячейка цилиндрической формы (длина $100 \mathrm{~mm}$,

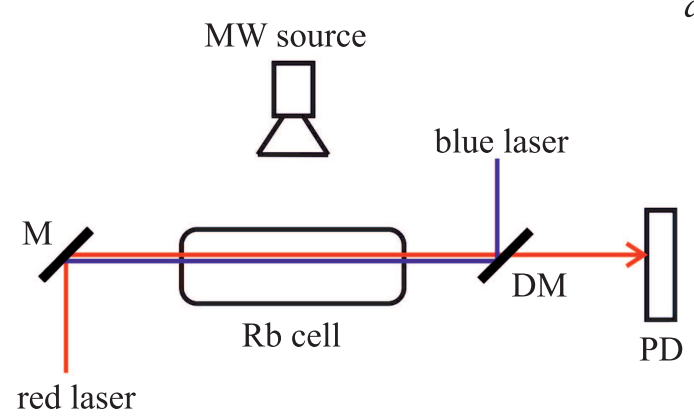

$a$

$b$

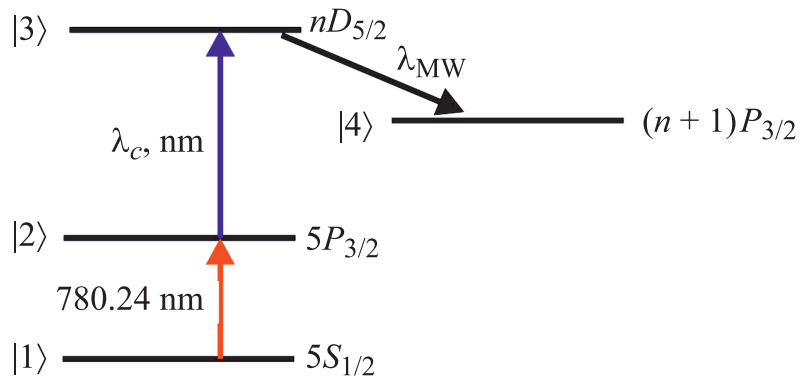

Рис. 1. $a-$ схема экспериментальной установки: СВЧ источник, пробный красный и связывающий синий лазеры, зеркало $\mathrm{M}$, дихроичное зеркало DM, ячейка, фотодетектор PD; $b-$ четырехуровневая энергетическая система для наблюдения расщепления АT в атомах Rb.

диаметр $25 \mathrm{~mm}$ ) с парами атомов $\mathrm{Rb}$ (естественная смесь изотопов ${ }^{85} \mathrm{Rb}$ и ${ }^{87} \mathrm{Rb}$ ), через которую проходили два противоположно направленных лазерных луча, сфокусированных в центре ячейки: пробный (красный) с длиной волны $780 \mathrm{~nm}$ и связывающий (синий) с перестраиваемой длиной волны $479-486 \mathrm{~nm}$ (рис. 1). СВЧ излучение направлялось перпендикулярно лазерным лучам. Существенные для данного метода атомные состояния $\mathrm{Rb}$ образуют систему четырех энергетических уровней: $5 S_{1 / 2}-5 P_{3 / 2}-n D_{5 / 2}-(n+1) P_{3 / 2}$.

Фотодетектор регистрирует сигнал пропускания пробного (красного) излучения, получаемого при сканировании частоты лазера по допплеровскому контуру, образуемому оптическими переходами $5 S_{1 / 2}-5 P_{3 / 2}$ атома ${ }^{85} \mathrm{Rb}$. Сканирование частоты пробного излучения осуществляется 10 Гц-пилообразной модуляцией тока инжекции красного лазера. При включении синего лазера в спектре появляется пик прозрачности (рис. 2). Данный эффект называется эффектом ЭИП [17]. Для измерения напряженности электрического поля на ячейку подается СВЧ излучение с частотой, равной частоте перехода $n D_{5 / 2}-(n+1) P_{3 / 2}$. Под действием этого поля происходит расщепление уровня энергии ридберговского состояния $n D_{5 / 2}$ на два подуровня (эффект AT [6]) с энергиями (1). При этом пик ЭИП расщепляется на две составляющие, разность частот между которыми $\Delta f_{\text {probe }}$ зависит от величины напряженности 


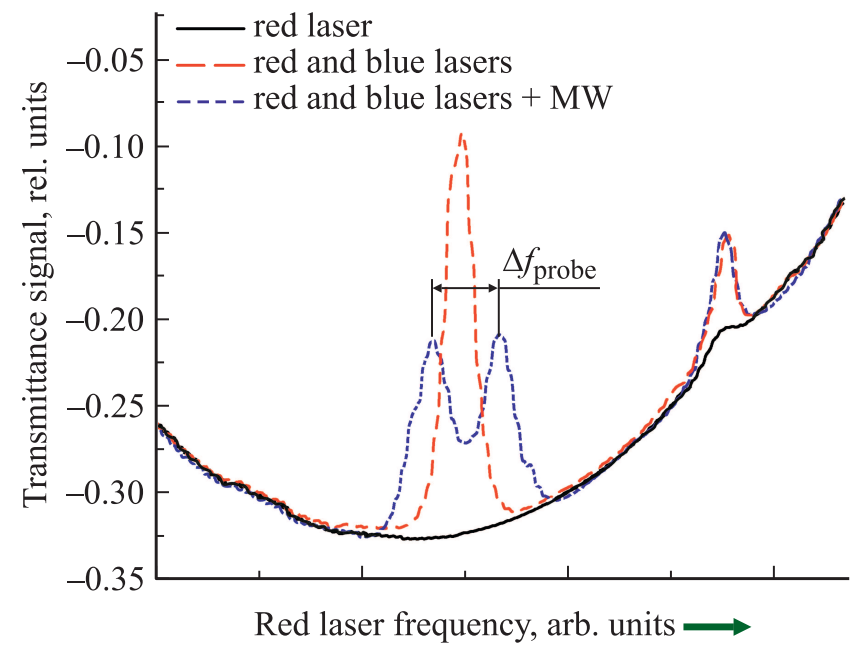

Рис. 2. Сигнал пропускания пробного света при сканировании частоты красного лазера по допплеровскому контуру, образуемому оптическими переходами $5 S_{1 / 2}-5 P_{3 / 2}$ атома ${ }^{85} \mathrm{Rb}$.

электрической составляющей прилагаемого СВЧ поля. Для вычисления напряженности электрического поля используется выражение (3). С учетом допплеровских сдвигов частот пробного (красного) и связывающего (синего) излучений выражение (3) приводится к виду

$$
E=\frac{\lambda_{p} \Delta f_{\text {probe }}}{\lambda_{c}|\mathscr{R}|} .
$$

Для генерации пробного излучения был выбран компактный DBR-лазер (лазер с распределенным брэгговским отражением) с длиной волны $780 \mathrm{~nm}$ и опцией модуляции тока инжекции лазера на частотах вплоть до $500 \mathrm{MHz}$. Генерация DBR-лазера при таких высоких частотах модуляции происходит без срыва одномодового режима генерации, что отличает данный тип лазеров от диодных лазеров с внешним резонатором. Спектр выходного модулированного излучения состоит из излучения на несущей частоте и излучений, соответствующих двум боковым составляющим первого порядка. При сканировании несущей частоты лазера все три составляющих его выходного излучения поочередно образуют резонансы ЭИП. Сканирование частоты с помощью пилообразной модуляции тока инжекции лазера определяет линейный по времени характер изменения его частоты, что позволяет с большим, субгерцовым разрешением масштабировать по частоте ось временной развертки осциллографа, на котором наблюдается сигнал детектированного пробного (красного) излучения. Чтобы измерить расщепление $\Delta f_{\text {probe }}$, использовалась дополнительная синусоидальная частотная модуляция тока инжекции данного лазера $F_{\text {mod }}=60 \mathrm{MHz}$. На рис. 3, $a$ изображена стандартная осциллограмма сигнала ЭИП. Вторая осциллограмма (рис. $3, b$ ) получена при частотной модуляции тока пробного лазера. Кроме центрального пика дополнительно появляются боковые. Разность частот между пиками, соответствующими центральному пику и одному из боковых, определяется частотой модуляции $F_{\text {mod }}$.

Контроль и точная настройка длины волны излучения связывающего (синего) лазера осуществлялась при помощи измерителя длин волн Angstrom WS-U2. Часть излучения этого лазера, представляющего собой диодный лазер с внешним резонатором на дифракционной решетке, заводится в измеритель длин волн, который формирует сигнал ошибки и отправляет его на блок частотной автоподстройки лазера. При настройке синего лазера на длину волны $480.13 \mathrm{~nm}$, что соответствует переходу $5 P_{3 / 2}(F=4)-50 D_{5 / 2}$, была получена зависимость величины расщепления пика прозрачности от корня из мощности $\sqrt{P}$ на выходе рупора СВЧ источника (рис. 4). Данный выбор длины волны синего лазера позволяет проводить измерения напряженности поля на частоте $17.04 \mathrm{GHz}$. Зависимость носит линейный характер, что подтверждает теорию $[1,7,8,10]$ и выражение (9).

В ходе измерений было выяснено, что величина расщепления $\Delta f_{\text {probe }}$ зависит от поперечного смещения
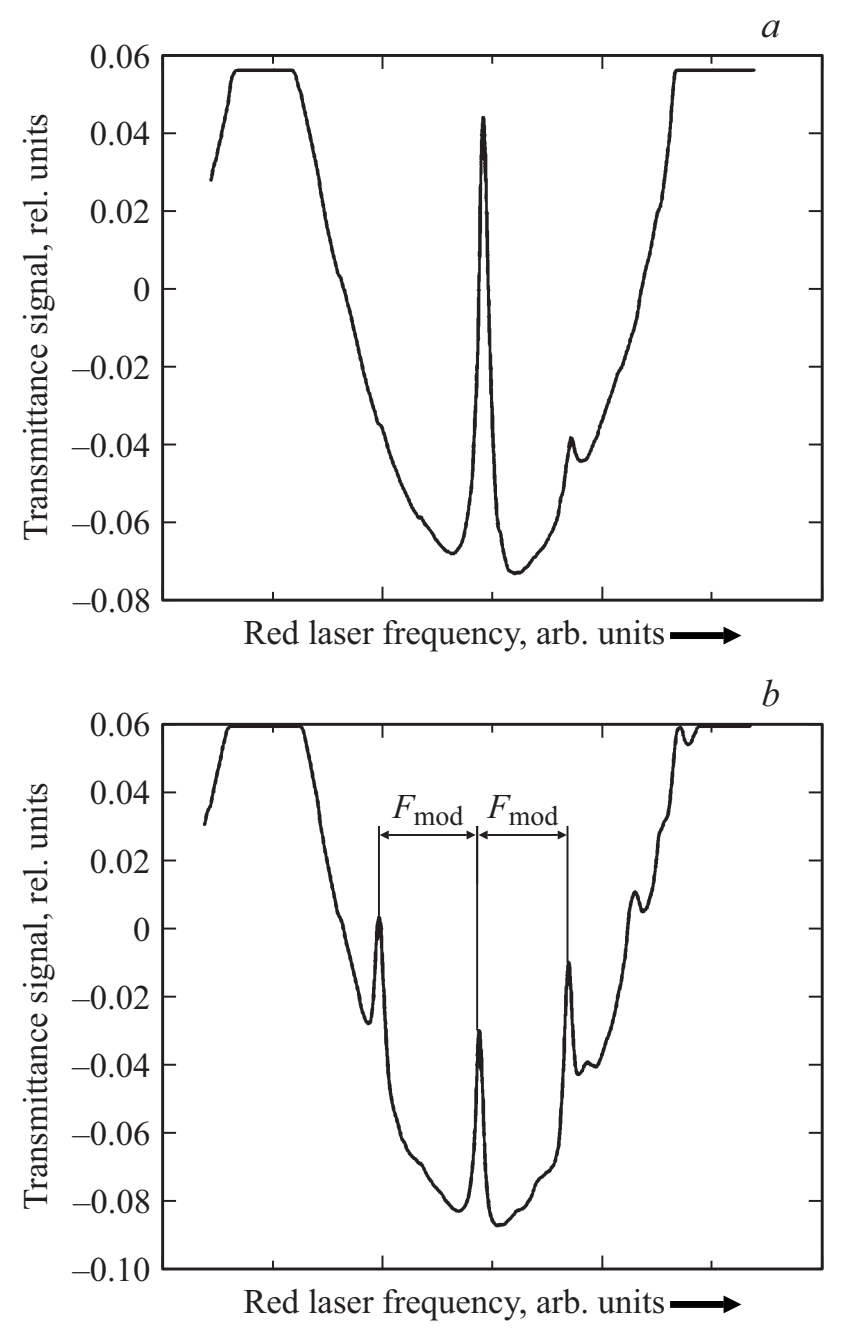

Рис. 3. Сигнал ЭИП без дополнительной частотной модуляции пробного лазера $(a)$, с дополнительной частотной модуляцией пробного лазера $(b)$. 


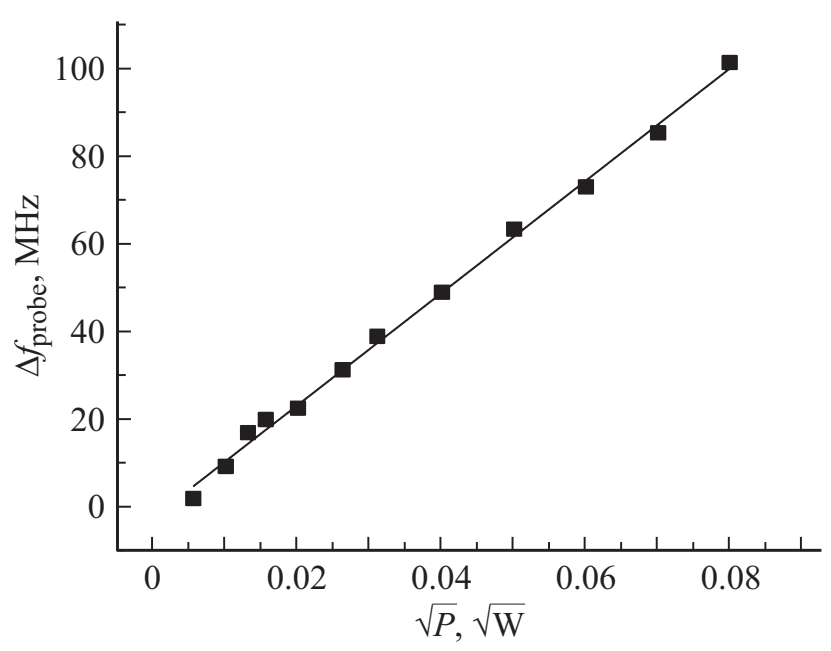

Рис. 4. Зависимость измеренного значения $\Delta f_{\text {probe }}$ (квадраты) от корня из мощности $\sqrt{P}$ на выходе рупора СВЧ источника и ее линейная аппроксимация.
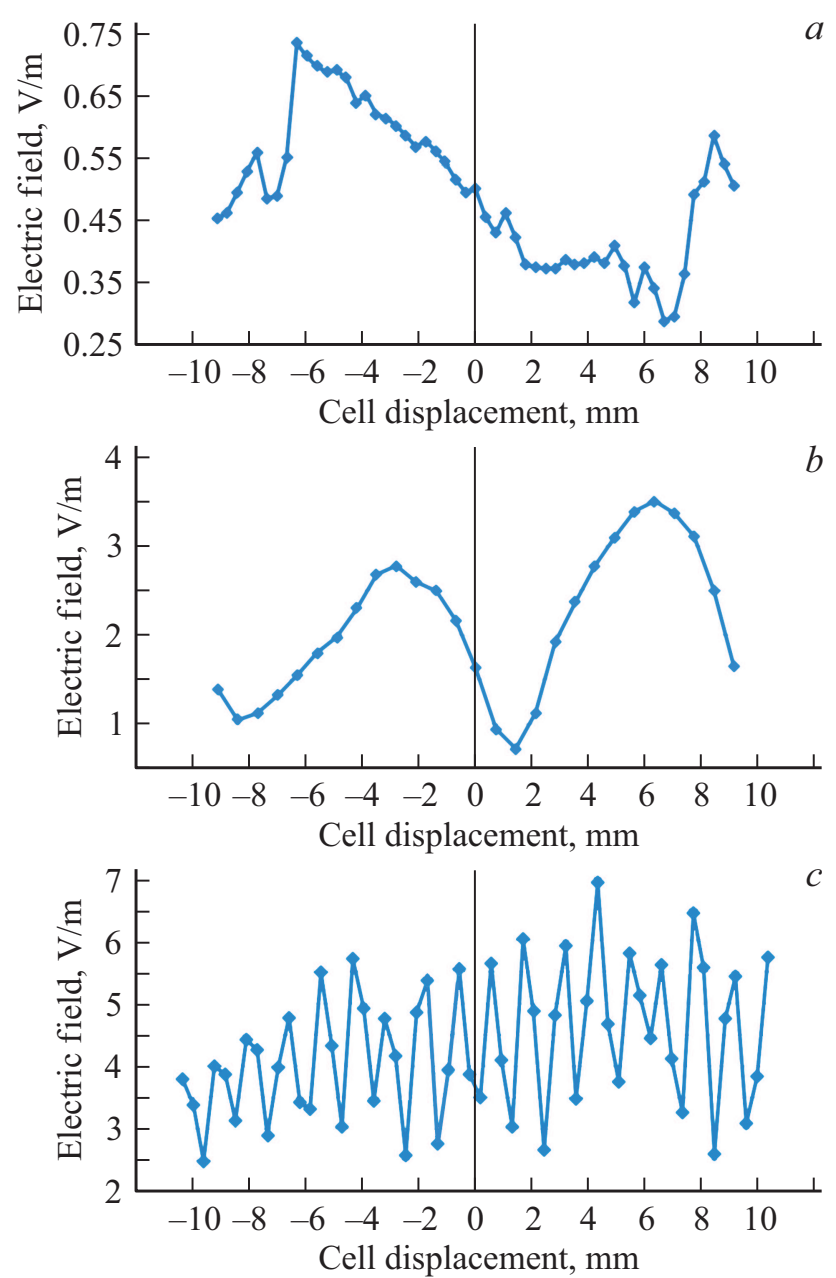

Рис. 5. Распределение напряженности электрического поля $E$ в зависимости от поперечного смещения ячейки. Частота СВЧ поля: $a-4.782 \mathrm{GHz}, b-17.04 \mathrm{GHz}, c-126.5 \mathrm{GHz}$.
Численные значения частот СВЧ поля $F_{M W}$, соответствующие им значения главных квантовых чисел $n$, длины волн синего лазера $\lambda_{c}$ и матричные элементы $\mathscr{R}$ дипольных переходов

\begin{tabular}{c|c|c|c|c}
\hline$F_{M W}, \mathrm{GHz}$ & $n$ & $\lambda_{c}, \mathrm{~nm}$ & $\mathscr{R}$, a.u. & $K, \mathrm{~V} /(\mathrm{mMHz})$ \\
\hline 2.10 & 99 & 479.378 & $6.297 \cdot 10^{3}$ & $2.02 \cdot 10^{-2}$ \\
4.78 & 76 & 479.488 & $3.682 \cdot 10^{3}$ & $3.45 \cdot 10^{-2}$ \\
17.04 & 50 & 480.125 & $1.566 \cdot 10^{3}$ & $8.11 \cdot 10^{-2}$ \\
126.50 & 26 & 483.222 & 402.9 & 0.313 \\
150.40 & 25 & 483.603 & 371.0 & 0.340 \\
196.55 & 23 & 484.490 & 311.0 & 0.405
\end{tabular}

ячейки в направлении, перпендикулярном прямой, по которой распространяются лазерные пучки. Так как значение длины волны СВЧ поля сравнимо с размерами стеклянной ячейки, генерируемая СВЧ источником падающая волна образует внутри ячейки стоячие волны. Соответственно получаемые значения напряженности электрического поля также будут отличаться друг от друга в зависимости от поперечного смещения ячейки с атомами. Ниже приведена таблица численных значений частот СВЧ поля $F_{M W}$, соответствующие им значения главных квантовых чисел $n$, длины волны синего лазера $\lambda_{c}$ и матричных элементов $\mathscr{R}$ дипольных переходо $n D_{5 / 2}-(n+1) P_{3 / 2}$, рассчитанных с помощью аппроксимационной формулы (8). По формуле (9) рассчитаны соответствующие значения напряженности электрического поля, которые можно описать выражением:

$$
E\left(n D_{5 / 2} \rightarrow(n+1) P_{3 / 2}\right)=K\left(\frac{V}{\mathrm{~m} \mathrm{MHz}}\right) \Delta f_{\text {probe }}(\mathrm{MHz}) .
$$

Для расчета коэффициента $K=\lambda_{p} /\left(\lambda_{c} \mathscr{R}\right)$ использованы численные значения длины волны пробного излучения $\lambda_{p}=780.24 \mathrm{~nm}$, приведенных в таблице длин волн $\lambda_{c}$ связывающего излучения, а также матричных элементов $\mathscr{R}$ соответствующих СВЧ переходов.

Для представленных в таблице частот СВЧ (микроволнового) излучения $F_{M W}$ были получены графики распределения напряженности электрического поля (НЭП) $E$ от положения ячейки (рис. $5, a, b, c)$.

Из полученных результатов следует, что поле внутри цилиндрической ячейки, внешние диаметр и длина которой равны соответственно 25 и $100 \mathrm{~mm}$, неоднородно. Характер неоднородности разный в трех случаях: когда диаметр $D$ ячейки меньше длины волны СВЧ поля (рис. $5, a$ ), сравним с длиной волны (рис. $5, b$ ) и больше длины волны (рис. $5, c$ ).

В первом случае (рис. 5, $a$ ), согласно [18], получено, что при $D / \lambda_{M W}=0.08$, где $D-$ размер ячейки, $\lambda_{M W}-$ длина волны СВЧ излучения, погрешность за счет отличия измеряемого поля от поля в полости ячейки, меньше чем $0.7 \%$. Утверждается, что в диапазоне частот СВЧ излучения от 10 до $30 \mathrm{GHz}$ при выполнении условия $D / \lambda_{M W}<0.1$ достигается точность, которую дают существующие методы измерения НЭП. 
Второй случай реализуется на частоте $17.04 \mathrm{GHz}$, когда $D / \lambda_{M W}=1.3$, т.е. когда геометрические размеры порядка длины волны и в ячейке укладывается несколько полуволн СВЧ излучения (рис. 5, $b$ ). Поправку на пространственное распределение рассеянного поля в ячейке можно получить расчетным путем с помощью программного продукта HFSS (high frequency structural simulator). Для оценки погрешности измерения НЭП необходимо предварительно получить связь между полем, рассеянным в ячейке, и падающим полем, которое принимается за эталонное. Другими словами, ячейка должна быть предварительно откалибрована по эталонному излучению. Погрешность измерения квантовым методом кроме погрешности, с которой известно эталонное поле, будет зависеть от формы калибровочной кривой, от дискретности с которой она снята экспериментально и т.д.

В третьем случае в ячейке укладывается много полуволн СВЧ излучения (рис. 5,c). Если допустить, что характер распределения минимумов и максимумов случайный, то тогда распределение поля может быть охарактеризовано средним значением и среднеквадратическим отклонением, с привязкой среднего значения к НЭП падающего поля.

Из проведенного анализа следует, что учесть влияние ячейки на результат измерения квантовым методом невозможно без знания эталонного поля, падающего на ячейку.

\section{Заключение}

В настоящей работе представлен теоретический и экспериментальный анализы эффекта расщепления оптического резонанса ЭИП в СВЧ поле, на основе которого разработан прибор для измерения напряженности электрического поля нового типа. В результате процесс измерения напряженности электрического поля СВЧ излучения переносится в область высокоточных измерений частоты оптических сигналов и не связан более с измерением электрических величин (тока, напряжения, мощности).

Ключевым элементом измерительного прибора становится атомарная среда, а именно помещенные в ячейку щелочные атомы, возбужденные до ридберговских состояний. Напряженность СВЧ электрического поля в ячейке определяется по измерению частоты расщепления ЭИП прозрачности с использованием рассчитанных значений атомного дипольного момента СВЧ перехода (8). Частота измеряется с помощью индикационной системы, конечным элементом которой в представленной работе являлся осциллограф. Представленный метод измерений обладает широкополосностью и линейностью. Проведено измерение НЭП на дискретных частотах 2.10, 4.78, 17.04, 126.50, 150.40 и $196.55 \mathrm{GHz}$.

Экспериментально показано, что размеры ячейки и электрические характеристики материала, из которого она изготовлена, оказывают существенное влияние на точность измерения СВЧ поля за счет возникающих резонансов во внутренней полости ячейки. Результат измерения НЭП зависит от поперечного смещения ячейки относительно линии, по которой направлены лазерные лучи. Учесть влияние конкретной ячейки на результат измерения невозможно без знания эталонного поля, падающего на ячейку, и ее предварительной калибровки по этому полю. Процесс калибровки может быть упрощен, и точность измерений НЭП повышена, если положение ячейки относительно линии распространения лазерных лучей зафиксировано. Этого можно достичь, например, путем подачи на ячейку лазерного излучения по сопряженным с ячейкой оптоволоконным кабелям. Пример такой конструкции приведен на рис. 3 в работе [8]. Для уменьшения влияния параметров ячейки на результаты измерений НЭП также представляется возможным и перспективным использование в качестве ячейки с атомами оптоволоконного кабеля с полой сердцевиной, куда заводятся пары щелочных металлов (hollow-core photonic crystal fibers) [19].

В заключение отметим работу [20], в которой рассмотренный выше эффект расщепления резонанса ЭИП использован для создания высокочувствительного приемника широкополосного СВЧ сигнала на частоте $10.22 \mathrm{GHz}$, соответствующей резонансу на переходе между ридберговскими состояниями атомов рубидия $59 D_{5 / 2}$ и $60 P_{3 / 2}$. Таким образом, потенциальные возможности практического применения радиационных переходов между высоковозбужденными ридберговскими состояниями атомов не ограничиваются рассмотренным в настоящей работе измерением напряженности электрического поля СВЧ излучения и могут быть использованы для кодирования и передачи информации.

Наряду с ридберговскими состояниями атомов рубидия высоковозбужденные состояния атомов других химических элементов, прежде всего щелочных металлов (литий, натрий, калий, цезий), могут рассматриваться в качестве перспективных кандидатов для применения в области прецизионной метрологии СВЧ излучения. Использование радиационных переходов между ридберговскими состояниями для разработки методов высокоскоростной и достоверной передачи информации с помощью излучения гигагерцового и терагерцового диапазонов частот также требуют дальнейших теоретических и экспериментальных исследований электродинамических характеристик высоковозбужденных атомов.

\section{Финансирование работы}

Настоящая работа поддержана грантом РФФИ № 1802-00053-a, Министерством образования и науки РФ в рамках Госзадания по проекту № 3.7514.2017/8.9, Министерством науки и высшего образования РФ в рамках Госзадания по проекту FZGU-2020-0035 (В.Д.О.), а также грантом Российского научного фонда № 19-7230014 (В.Г.П.). 


\section{Конфликт интересов}

Авторы заявляют, что у них нет конфликта интересов.

\section{Список литературы}

[1] Holloway C.L., Gordon J.A., Jefferts S., Schwarzkopf A., Anderson D.A., Miller S.A., Thaicharoen N., Raithel G. // IEEE Trans. on Antennas and Propagation. 2014. V. 62. P. 6169.

[2] Holloway C.L., Simons M.T., Gordon J.A., Wilson P.F., Cooke C.M., Anderson D.A., Raithel G. // IEEE Trans. Electromagn. Compat. 2017. V. 59. P. 717-728.

[3] Anderson D.A., Raithel G. // Phys. Lett. 2017. V. 111. P. 053504.

[4] Kumar S., Fan H.Q., Kübler H., Sheng J.T., Shaffer J.P. // Sci. Rep. 2017. V. 7. P. 42981.

[5] Jiao Y.C., Hao L.P., Han X.X., Bai S.Y., Raithel G., Zhao J.M., Jia S.T. // Phys. Rev. Appl. 2017. V. 8. P. 14028.

[6] Autler S.H., Townes C.H. // Phys. Rev. 1955. V. 100. P. 703. Cohen-Tannoudji C.N. // Amazing Light / Ed. by Chiao R.Y. New York: Springer, 1996.

[7] Sedlacek J.A., Schwettmann A., Kübler H., Löw R., Pfau T., Shaffer J.P. // Nature Phys. 2012. V. 8. P. 819.

[8] Simons M.T., Gordon J.A., Holloway C.L. // Appl. Opt. 2018. V. 57. N 22. P. 6456.

[9] Manakov N.L., Ovsiannikov V.D., Rapoport L.P. // Phys. Rep. 1986. V. 141. P. 319.

[10] Mohapatra A.K., Jackson T.R., Adams C.S. //. Phys. Rev. Lett. 2007. V. 98. P. 113003.

[11] Собельман И.И. Введение в теорию атомных спектров. М.: Наука, 1977.

[12] Варшалович Д.А., Москалев А.Н., Херсонский В.К. Квантовая теория углового момента. Л.: Наука, 1975.

[13] Ильинова Е.Ю., Овсянников В.Д. // ОПт. и спектр. 2008. T. 105. № 5. C. 709-719.

[14] Бейтмен Г., Эрдейи А. Высшие трансцендентные функции. Т. 1. М.: Наука, 1973. 295 с.; Т. 2. М.: Наука, 1974. $296 \mathrm{c}$.

[15] Ralchenko Yu., Kramida A., Reader J., NIST ASD Team. NIST Atomic Spectra Database (version 4.1), [Online]. National Institute of Standards and Technology, Gaithersburg, MD [Электронный ресурс] Режим доступа: http://physics.nist.gov/asd

[16] Информационная система „Электронная структура атомов“. Новосибирский государственный университет. Институт автоматики и электрометрии СО РАН. [Электронный ресурс] Режим доступа: http:/grotrian.nsu.ru

[17] Boller K.-J., Imamoğlu A., Harris S.E. // Phys. Rev. Lett. 1991. V. 66. P. 2593.

[18] Fan H., Kumar S., Sheng J., Shaffer J.P., Dodge H.L., Holloway C.L., Gordon J.A. // Phys. Rev. Appl. 2015. V. 4. P. 044015.

[19] Benabid F., Couny F., Knight J.C., Birks T.A., Russell P.S. // Nature. 2005. V. 434. N 7032. P. 488.

[20] Song Z., Liu H., Liu X. et al. // Opt. Express. 2019. V. 27. N 6. P. 8848. 